\title{
Introducing Full-Aperture FJP (faFJP), a Technique for Manufacturing Sub-Millimetre Aspheres
}

\author{
W.A.C.M. Messelink ${ }^{1,2, *}$ and M. Meeder ${ }^{2,3}$ \\ ${ }^{1}$ Edmund Optics Singapore, Singapore, 738100, Singapore \\ ${ }^{2}$ Fisba AG, Sankt Gallen, CH-9016, Switzerland \\ ${ }^{3}$ ETH Zürich, Zürich, CH- 8092, Switzerland
}

\begin{abstract}
We propose a novel approach, "faFJP", to the computer controlled polishing (CCP) of mini aspheres down to $0.2 \mathrm{~mm}$. The generation of locallytunable, full-aperture footprints is accomplished by applying a Fluid Jet Polishing (FJP) beam larger than the full aperture being polished and varying the incident angle.
\end{abstract}

\section{Introduction}

Thanks to their point symmetry, spherical lenses have been produced for centuries in high qualities and quantities, with shape deviations down to dozens of nanometres, by applying chaotic, semi-random tool paths above the rotating workpiece. Unfortunately, optics designers have been insisting on using aspherical lenses, due to their better performance in handling optical rays and because the use of one of these types of lenses replaces up to five spherical lenses within the design of an optical system. The fact that aspherical lenses possess line symmetry, rather than point symmetries, forced optical fabrication engineers to invent numerous dedicated polishing techniques, each featuring the generation and application of sub-aperture footprints (local polishing spots within the clear aperture of the optical surface) such as belts [1], bonnets [2], fluid jets [3, 4], ion beams [5], plasma jets [6], magnetorheological fluids [7], rotating spheres [8] or polyurethane needles. All these techniques distinguish themselves in the type of aspherical shapes producible, quality generable or dimensions manageable. In this paper, we propose an alternative approach, named "faFJP", to the computer controlled polishing (CCP) of mini aspheres featuring clear-apertures down to $0.2 \mathrm{~mm}$. Instead of traversing a static, sub-aperture footprint across the clear-aperture, faFJP uses a locally-tunable, full-aperture footprint by applying a macroscopic Fluid Jet Polishing (FJP) beam, which has a diameter larger than the clear aperture being polished. The variation of the footprints is generated by changing the incident angle of the fluid beam with respect to the asphere axis of symmetry, due to FJP's characteristic wear profile.

\section{Theory and simulations}

Finnie et al. [9] established the dependency of the volumetric removal on the angle of incidence of an abrasive particle. Particularly it was shown that the highest removal did not occur

\footnotetext{
*e-mail: PMesselink@edmundoptics.com.sg
} 

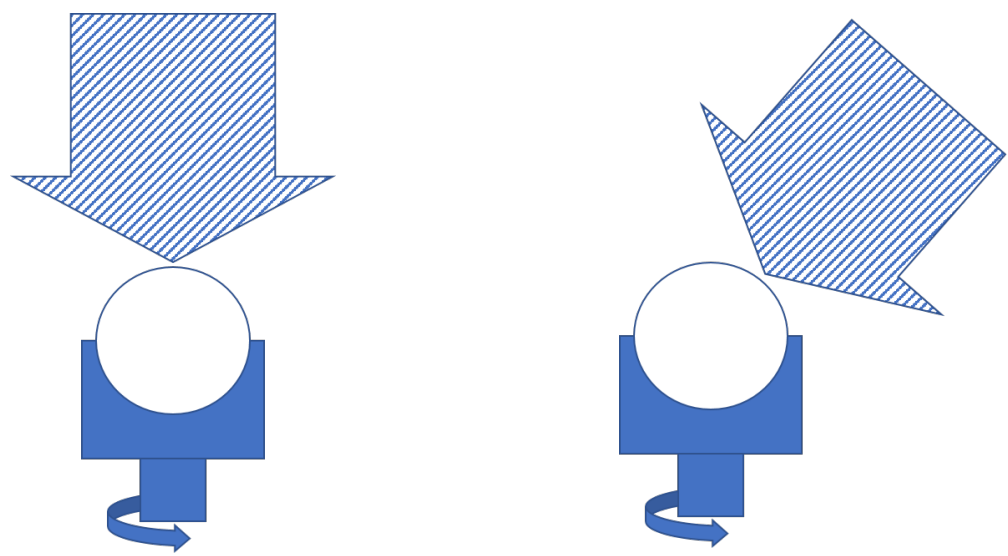

Figure 1: Diagrams of a macroscopic FJP beam, impinging on a spherical substrate with a diameter smaller than the width of the beam, at different angles.

when the particle impacted the surface perpendicularly, but at a specific grazing incidence. For a macroscopic FJP beam, impinging on a spherical substrate with a diameter smaller than the width of the beam (see Figure 1), this results in a rotationally symmetric removal profile, with the highest removal some distance away from the centreline of the beam. Simulations of the removal profiles for different angles of the FJP beam while the workpiece is not rotating are shown in Figure 2. If the workpiece is mounted on a rotary table and spun
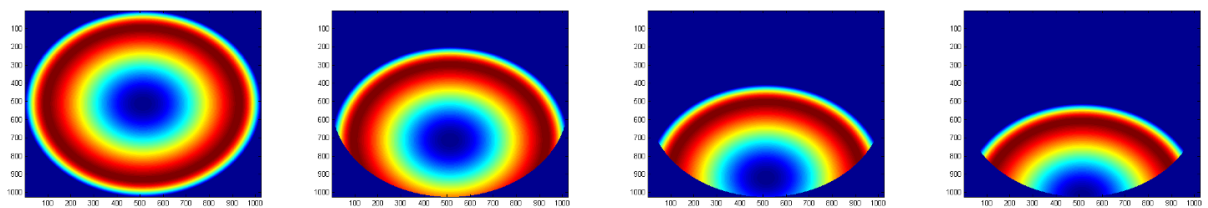

Figure 2: Simulations of the removal profiles for different angles of the FJP beam on a nonrotating workpiece.

underneath the FJP beam, then the removal profile will be distributed tangentially over the surface. A simulation of this for different angles of the FJP beam is shown in Figure 3. Each
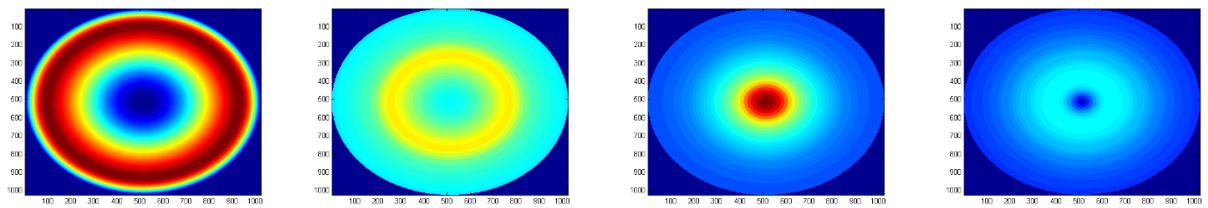

Figure 3: Simulations of the removal profiles for different angles of the FJP beam on a rotating workpiece.

of the removal profiles from Figure 3 produces an asphere. Increasing the time the workpiece 
spends inside the FJP beam increases the aspheric departure that is generated. And by using a linear combination of (beam) angles and (dwell) times an arbitrary aspheric profile can be generated.

\section{Conclusions and future work}

We have introduced a novel method of generating and correcting mini aspheres that applies full-aperture, but locally-tunable footprints. As opposed to using a static, sub-aperture footprint that is traversed over the full-aperture, as is the state of the art for macro aspheres. The generation of locally-tunable, full-aperture footprints is accomplished by applying an FJP beam larger than the full aperture being polished and varying the incident angle. The theory of volumetric removal dependency on the angle of incidence has been introduced and the effect on a spherical surface in a macroscopic FJP beam has been derived and simulated.

Arbitrary asphere profiles can be generated by applying a linear combination of FJP beam angles. In practice, this linear combination can be implemented by mounting the FJP beam on a pivot and placing the workpiece in the centre of the pivot. By rotating the pivot, the different FJP beam angles can be obtained sequentially and by varying the speed of rotation the required dwell times for each angle can be achieved.

The next step is to experimentally verify the introduced technique. Future research could examine the orthogonality or joint entropy of the removal profiles for different angles to assess what shapes of aspheres are more straightforward to generate.

\section{Acknowledgement}

The theoretical work presented in this paper was performed while all the authors were employed by Fisba AG, Sankt Gallen, CH-9016, Switzerland. We would especially like to thank Dr. Oliver Faehnle for his guidance and useful discussions.

\section{References}

[1] E. Fess, J. Schoen, M. Bechtold, D. Mohring, UltraForm finishing, in Window and Dome Technologies and Materials IX, edited by R.W. Tustison (SPIE, 2005), Vol. 5786 of Proc. SPIE, pp. 305-309, http://dx.doi.org/10.1117/12.606468

[2] D.D. Walker, D. Brooks, R. Freeman, A. King, G. McCavana, R. Morton, D. Riley, J. Simms, First aspheric form and texture results from a production machine embodying the precession process, in Optical Manufacturing and Testing IV, edited by H.P. Stahl (2001), Vol. 4451 of Proc. SPIE, pp. 267-276, http://dx.doi.org/10.1117/12. 453652

[3] O.W. Faehnle, H. van Brug, H.J. Frankena, Applied Optics 37, 6771 (1998)

[4] W.A. Messelink, O.W. Faehnle, Exploiting the Process Stability of Fluid Jet Polishing, in Frontiers in Optics 2008/Laser Science XXIV/Plasmonics and Metamaterials/Optical Fabrication and Testing (Optical Society of America, 2008), p. OThD3, http://www . opticsinfobase. org/abstract . cfm?URI=0FT-2008-0ThD3

[5] S. Zafran, K.A. Kaufman, M.M. Silver, Optical Engineering 21, 216002 (1982)

[6] T. Arnold, G. Boehm, I.M. Eichentopf, M. Janietz, J. Meister, A. Schindler, Vakuum in Forschung und Praxis 22, 10 (2010)

[7] S.D. Jacobs, D. Golini, Y. Hsu, B.E. Puchebner, D. Strafford, I.V. Prokhorov, E.M. Fess, D. Pietrowski, W.I. Kordonski, Magnetorheological finishing: a deterministic process for optics manufacturing, in International Conference on Optical Fabrication and Testing, 
edited by T. Kasai (SPIE, 1995), Vol. 2576 of Proc. SPIE, pp. 372-382, http://dx. doi.org/10.1117/12.215617

[8] T. Hirata, Y. Takei, H. Mimura, Procedia CIRP 13, 198 (2014), 2nd CIRP Conference on Surface Integrity (CSI)

[9] I. Finnie, Wear 3, 87 (1960) 